\title{
Research on Semiconductor Refrigeration System with Current Adaptive Temperature
}

\author{
Xiaolin Zhang \\ Xinyu University, Xinyu, Jiangxi, China, 338004
}

\begin{abstract}
Keywords: Semiconductor Refrigeration Chip, Current, Adaptive Temperature, Improve the Cooling Performance
\end{abstract}

\begin{abstract}
According to the semiconductor refrigeration chip operating characteristic curve, it can be seen that the maximum cooling capacity and the maximum cooling efficiency corresponding to the working current is inconsistent and the current semiconductor refrigeration systems are a constant current and work, it is impossible to make it in the best working condition. In order to improve the performance of semiconductor refrigeration, this paper designs a current adaptive temperature control system, which automatically adjusts the current of the cooling chip according to the temperature of the cold and cold end face of the semiconductor cooling piece, so that it is always in the best working condition. Practice has proved that the system can not only improve the semiconductor cooling performance, but also effective management of semiconductor thermal power to improve the life of semiconductor chiller.
\end{abstract}

\section{Introduction}

Semiconductor refrigeration is a kind of thermoelectric refrigeration, is based on the Peltier effect on the basis of artificial refrigeration technology. Semiconductor refrigeration is due to no refrigerant, no noise, the small size of the refrigerator and widely used in electronics, medical, defense and other fields. But because the low efficiency of semiconductor refrigeration, it is about compression refrigeration efficiency of $1 / 3$, so that its development is subject to certain restrictions. Semiconductor cooling performance is mainly reflected in the cooling capacity and cooling efficiency of two aspects, affecting its performance in addition to the main factors of its own thermoelectric properties, the other important factor is the semiconductor cooling chip operating current. The cooling performance of the conventional semiconductor refrigerator is not optimal because the current between the maximum cooling capacity of the semiconductor refrigerator and the maximum cooling efficiency is different, and the current semiconductor coolers are operated at a constant current.

At present, the semiconductor cooler work, in order to meet the needs of rapid cooling, take a larger cooling capacity of working conditions. According to the working characteristics of the cooling chip, we can see that the current is between 4-6.5A and the semiconductor cooling capacity $\mathrm{Q}$ is large, Therefore, the existing semiconductor cooler is not only the cooling efficiency is low, and the hot side of the heat, there may be due to heat dissipation is not timely and burned half. 


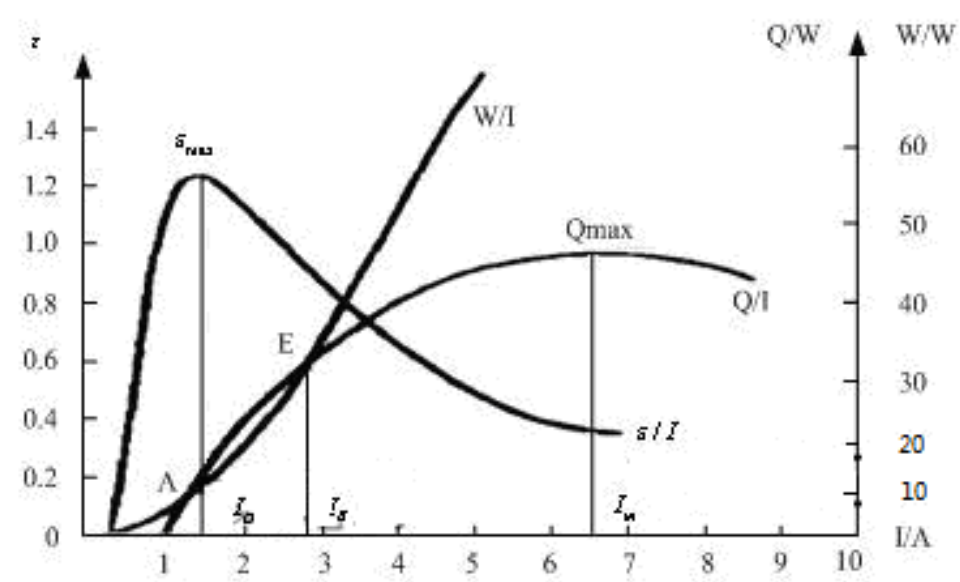

Figure 1 TEC refrigeration operating characteristics curve

In order to improve the performance of semiconductor chiller, this paper designs a control system with current change with temperature, automatically adjusts the size of semiconductor working current and adapts the current working condition so that the semiconductor chiller is always in the best working condition to meet the requirements of rapid cooling, But also reduce the purpose of energy consumption.

\section{The Structure and Principle of Semiconductor Cooling Fuzzy Control System}

The Principles of Semiconductor Refrigeration. According to the Peltier effect, a P-type semiconductor element and an N-type semiconductor components into a thermocouple, connected to the DC power supply at the junction will produce temperature and heat transfer. At the top of the joint, the current direction is N P, the temperature drops and absorbs heat, which becomes the cold end. At the bottom of the joint, the current direction is P N, the temperature rises and exotherms, which becomes the hot end. As shown in Fig. 1, the electric power, the cooling capacity and the cooling efficiency of the semiconductor cooling chip are expressed by $\mathrm{W}, \mathrm{Q}$, and $\varepsilon$, respectively, and the operating current Imo corresponding to the maximum cooling capacity Qmax corresponding to the maximum cooling capacity Qmax. The cooling capacity $\mathrm{Q}$, the cooling efficiency $\varepsilon$, and the consumed electric power $\mathrm{W}$ are the functions of the current I and the temperature difference $\Delta \mathrm{T}$. Q, $\mathrm{W}$ and $\varepsilon$ are only a function of the current $\mathrm{I}$ at a given temperature difference $\Delta \mathrm{T}$. Since the maximum cooling capacity and the maximum cooling efficiency of the semiconductor refrigeration chip correspond to the operating current, and with the semiconductor cooler operating conditions change. At present, the semiconductor cooler in accordance with the higher cooling capacity to determine the working current to meet the needs of rapid cooling, but led to the semiconductor refrigerator cooling efficiency is low, the shortcomings of large power consumption.

Principle of Current Adaptive Temperature Control System. As the semiconductor cooler is running, the cold junction temperature and the thermal side temperature difference of the semiconductor refrigeration chip are changed, which requires the semiconductor refrigeration chip operating current changes with the semiconductor cooler conditions change. When the semiconductor cooler box temperature and set the temperature difference between the hope that the semiconductor cooling chip in the maximum cooling capacity of the working state, and when the semiconductor refrigerator temperature and set the temperature close to the stable operation, the semiconductor refrigeration chip is expected to be the largest cooling efficiency working state, this must be based on changes in the semiconductor cooler working conditions in real time to adjust the current to achieve this purpose.

Since the maximum current corresponding to the cooling current Im and the maximum cooling efficiency corresponding to the current Io difference is large, if only adjustable constant current source to adjust, then the use of adjustable constant current source is damage. In this paper, we design a control system which integrates the adjustable constant current source from the adaptive adjustment and the serial connection of the semiconductor refrigeration chip, so that the current can 
be changed from Im to Io in a wide range, so that the semiconductor cooler is always in the most Good working condition.

It shows the structure of the semiconductor refrigeration control system with current self-adapting temperature. In the figure, the single-chip microcomputer is used as the processing center to receive the feedback signal of the cold and hot-side temperature sensors and the set temperature in the box. After the internal fuzzy algorithm is processed by the single-chip microcomputer, the output voltage is adjusted or the semiconductor string-parallel mode is changed to realize the automatic adjustment of the semiconductor refrigeration chip current process.

The Composition of the Main Hardware. The single-chip selection STC90C516RD +. SCM occupies a small space, affordable, but powerful. Can receive the temperature sensor signal, display the temperature, and compared with the set value, through the microcontroller I / O port control drive circuit.

The semiconductor cooler, including cooling film TEC-12706, cooling radiator, heat conduction module, cooling fan, cold end fan, insulation cotton, thermal grease and so on. Semiconductor cooler in the installation, the need to pay attention to the following points: the text of the semiconductor cooling film is the cooling surface, the need for a small chill block; no words of the semiconductor cooling film for the heating surface, the need for large radiators and cooling fans; Semiconductor cooling film on both sides need to be coated with silicone grease; semiconductor cooling chip connection line in the red line then positive, black line then negative.

The temperature sensor is using the DS18B20. Between the sensors and the microcontrollers is only one data line to communicate, that is, single-bus communication mechanism. Each sensor has a separate 64-bit serial number, located in the sensor's internal memory, when there are multiple sensors deployed in different locations, the microcontroller in the collection temperature, you can read the serial number to distinguish the way the sensor.

\section{The Design of Key Technology}

The Design of Automatic Adjustable Constant Current Source. Adjustable constant current source controller generally contains the oscillator, error amplifier, PWM flip-flop, state controller, high-quality high-power switch, current comparator and a variety of functional protection circuit, including PWM flip-flop Contains a pulse width adjustment potentiometer, this article will automatically control the microcontroller pulse width adjustable potentiometer, in order to achieve automatic adjustment of voltage and current.

The Series and Parallel Automatic Switching. Automatic adjustable constant current source adjustment range is controlled within $\pm 30 \%$, otherwise it may be because the duty cycle is too small to drive the load. The maximum cooling capacity of the semiconductor cooler corresponds to a maximum current equivalent to the maximum cooling efficiency of more than $\pm 30 \%$. Therefore, such a wide range of current regulation cannot be accomplished only through an adjustable constant current source. In this paper, the design of the relay through the single-chip, automatically change the semiconductor cooling chip within the serial and parallel connection and automatic adjustable constant current source combination of ways to get a wide range of precision current automatic adjustment.

\section{The Refrigeration Test}

Automatic adjustable constant current source and serial-parallel automatic switching combined with the semiconductor refrigeration system for sealed box cooling experiments, the experimental ambient temperature of 25 degrees Celsius and the cabinet temperature of 10 degrees Celsius.

The Rapid Cooling Stage. In order to simplify the fuzzy refrigeration control system, improve the current constant and fuzzy control system using non-continuous adjustment cooling stage is divided into three stages, respectively, the output of different current. At the beginning of the box temperature of 25 degrees Celsius, the need for rapid cooling, through the semiconductor cooling chip current of 5.1A, cooling chip cooling capacity of the largest; when the temperature inside the 
box down to 15 degrees Celsius, through the semiconductor cooling chip current automatically adjusted to 2.5A, the semiconductor cooling capacity is reduced, and the cooling efficiency is increased.

The Insulation Stage. When the temperature is 11 degrees Celsius, through the semiconductor cooling chip current automatically adjusted to $1.4 \mathrm{~A}$, then the semiconductor refrigeration chip cooling efficiency is highest, in the most power-saving state. After reaching the set temperature, the semiconductor cooling chip automatically power off, in the insulation state. The current in the semiconductor cooling chip in the insulated state will switch between 0 and 1.4A.

The Test Results Analysis. Through the semiconductor refrigeration test, we can learn that the current temperature of the semiconductor refrigeration system to enhance the semiconductor refrigeration performance is mainly reflected in the insulation phase. The main parameters are as follows:

(1) $70 \%$ of the current change range: the constant current system insulation phase between 0-5.1A switch; and current adaptive temperature control system insulation phase of the current between $0-1.4 \mathrm{~A}$ switch, the change range only constant Flow system of $27.5 \%$.

(2) $50 \%$ of the current change frequency: constant current system insulation time working time 386 seconds, downtime 765 seconds; and current adaptive temperature control system working time 955 seconds, downtime 1002 seconds. The same time, the current adaptive temperature control system to start less than half the number of constant current system start.

(3) the maximum cooling efficiency of the time greatly increased: constant current system operating current has been deviated from the maximum cooling efficiency of the corresponding current; and current adaptive temperature control system all the working hours of the insulation time in the maximum cooling efficiency.

\section{Conclusion}

Current adaptive temperature semiconductor refrigeration system, in the initial stage of work in the maximum cooling capacity of the state; and in the insulation phase of the work in the maximum cooling efficiency, so that the semiconductor cooling is always in the best working condition. Therefore, the current temperature of the semiconductor refrigeration system to optimize the working state, improve the cooling performance.

\section{Acknowledgements}

Fund Project: Research on enhancing the semiconductor refrigeration performance based on the fuzzy PID algorithm, Xinyu City Science and Technology Bureau Funded Projects (20163090864).

\section{References}

[1] Li Aibo. Single-stage semiconductor cooler refrigeration characteristics analysis and research [D]. Wuhan: Huazhong University of Science and Technology, 2011

[2] Yang Xiaoling. High precision temperature control system based on semiconductor refrigeration [J]. Instrument Technique and Sensor, 2007.3

[3] Li Hang, Liao Guipan, Wang Yilong, Liu Zhanyu. Small temperature control system based on semiconductor refrigeration device [J]. Automation letter, 2008.11

[4] Liu Ming, Cheng Youkai. Optimization of semiconductor refrigeration performance[J]. Energy Conservation, 2008 (3): 12-15

[5] Xie Ling. Semiconductor refrigeration technology development and application [J]. Clean and air-conditioning technology, 2008.1

[6] Li Lixin, Ji Jianguang, $\mathrm{Ni} \mathrm{Hai}$ et al. Simulation and regulation of semiconductor refrigeration-heating chamber [J]. Fluid Machinery. 2006, 34 (11): 37-71 